\title{
Recent advances and perspectives on space-time coding digital metasurfaces
}

\author{
Lei Zhang ${ }^{1}$, Jun Yan Dai ${ }^{1}$, Massimo Moccia ${ }^{2}$, Giuseppe Castaldi ${ }^{2}$, Tie Jun Cui ${ }^{1,{ }^{*}}$, and Vincenzo Galdi ${ }^{1,{ }^{* *}}$ \\ ${ }^{1}$ State Key Laboratory of Millimeter Waves, Southeast University, Nanjing 210096, PR China \\ ${ }^{2}$ Fields \& Waves Lab, Department of Engineering, University of Sannio, Corso Garibaldi 107, 82100, Benevento, Italy
}

Received: 14 October 2020 / Accepted: 13 November 2020

\begin{abstract}
Within the overarching framework of space-time metastructures, digital metasurfaces based on spatio-temporal coding are emerging as powerful and versatile architectures for complex field manipulations, also in view of their inherently programmable nature. Here, we provide a compact survey of our recent results and ongoing studies in this research area. Examples of field manipulations include harmonic beam steering and/ or shaping and programmable nonreciprocal effects. Possible applications are abundant and range from wireless communications to radars and imaging.
\end{abstract}

Keywords: Metasurfaces / space-time / digital / coding / communications / radars

\section{Introduction}

The concept of "digital" metastructures was put forward in 2014, independently, by the Groups led by Profs. Della Giovampaola and Engheta [1] and Cui et al. [2]. The basic idea underpinning these types of artificial materials is the reliance on a limited number of elementary inclusions (in the most extreme case, only two), and yet the ability to engineer a variety of complex field manipulations. Whenever applicable, this approach greatly simplifies the design process, as the search space can be effectively explored via discrete-optimization strategies [3]. Moreover, it streamlines the fabrication process, also improving the robustness with respect to the unavoidable tolerances.

Referring to an electromagnetic (EM) metasurface scenario [2], one may think of a set of reflecting elements, such as metallic patches laid on a grounded dielectric substrate, ideally characterized by a unit-amplitude reflection coefficient and a quantized phase response that can be univocally associated with a digital coding, for example, $0^{\circ}$ and $180^{\circ}$ in the simplest binary case, $0^{\circ}$, $90^{\circ}, 180^{\circ}$ and $270^{\circ}$ in a 2 -bit case, and so on. In this way, each possible spatial combination of these elements can be equivalently described in terms of a digital coding sequence. To some extent, this concept can also be interpreted as a generalization of the so-called "checkerboard" metasurfaces, featuring periodic distributions of metallic and artificial-magnetic-conductor elements [4].

\footnotetext{
* e-mail: tjcui@seu.edu.cn

** e-mail: vgaldi@unisannio.it
}

The advantages of a digital coding representation become even more evident in the presence of reconfigurable elements which, via the integration of active elements such as diodes or micro-electro-mechanical systems, can implement all required states. In this way, by controlling independently the coding elements via an electronic circuit such as a field-programmable gate array (FPGA), it is possible to attain a completely "programmable" metasurface platform [2]. In such scenario, the digital coding sequence defines a desired field manipulation, in analogy with a software application defining a desired functionality in a computer or a portable electronic device.

This is an extremely fascinating concept, with a wealth of interesting implications and ramifications, which has found a broad variety of applications. At microwave frequencies, particularly worth of mention are those to dynamical beam/polarization manipulations [5,6], reprogrammable holograms [7] and imaging [8], independent control of co-polarized amplitude and phase responses [9], scattering-signature reduction $[10,11]$, and direct transmission of digital messages [12]. Applications to terahertz frequencies have also been actively pursued [13], as well as transmission-type [14], transmission-reflection-integrated [15], and acoustic [16] scenarios. Perhaps, the most thought-provoking aspect of digital coding metastructures is the bridging between wave physics and information science $[17,18]$. This leads to a grand vision of "information metastructures" which leverages the programmable nature to implement software-defined, self-adaptive, and cognitive functionalities, possibly powered by artificial intelligence $[19,20]$. 
In conventional digital coding metasurfaces, the coding sequence is typically fixed in time and changed only whenever there is a need to switch among different functionalities. However, with the mounting interest in space-time metastructures [21-24] characterized by spatially and temporally variant properties, it makes sense to exploit the temporal dimension as well, by applying a dynamic switching of the coding sequence. This is the key idea behind space-time coding digital metasurfaces [25], which extend and generalize the concepts of timemodulated arrays [26] and phase-switched screens $[27,28]$, and have been successfully applied to several scenarios including harmonic beam steering and shaping [29], nonreciprocal effects [30], harmonic manipulations [31-33], scattering-signature control [34], wireless communications [35,36], and analog computing [37].

In what follows, we provide a survey of our previous results and ongoing investigations in this fast-pacing research area. Accordingly, starting from the basic concepts and related theoretical aspects (Sect. 2), we then illustrate some recent advances and applications (Sect. 3), and finally discuss the challenges and perspectives (Sect. 4).

\section{Basic idea and theory of space-time coding digital metasurfaces}

Referring to [25] for more details, we briefly summarize the concept of space-time coding digital metasurfaces, which is schematized in Figure 1a. We consider a digital, programmable metasurface comprising a 2-D arrangement of $M \times N$ identical elements (yellow patches) whose reflection coefficients can be controlled electronically via positiveintrinsic-negative (PIN) diodes so as to assume a unit amplitude and a set of quantized phases. In the simplest binary case, the response is switched via a diode between two possible states (e.g., in-phase and out-of-phase reflection), associated with the $0 / 1$ bits. We assume that the switching is controlled in space and time by an FPGA, according to a given coding (represented by the 3-D matrix of red and green dots).

In [25], an approximate analytical model was put forward for the scattered field due to a normally incident time-harmonic illumination with $\exp \left(j 2 \pi f_{c} t_{c}\right)$ dependence. Such model essentially relies on an adiabatic approximation of the physical-optics model developed in [2] for conventional (time-invariant) digital coding metasurfaces, and holds for modulation frequencies much smaller than the EM frequency $f_{c}[38,39]$. Accordingly, the farfield time-domain scattered field is expressed as

$$
\begin{aligned}
& f(\theta, \varphi, t) \approx E(\theta, \varphi) \sum_{q=1}^{N} \sum_{p=1}^{M} \Gamma_{p q}(t) \\
& \quad \times \exp \left\{j \frac{2 \pi}{\lambda_{c}} \sin \theta\left[(p-1) d_{x} \cos \varphi+(q-1) d_{y} \sin \varphi\right]\right\},
\end{aligned}
$$

with $E(\theta, \varphi)$ denoting the coding-element far-field pattern at the center frequency $f_{c}, \theta$ and $\varphi$ the conventional spherical angles, $d_{x}$ and $d_{y}$ the inter-element spacings along the $x$ and $y$ directions, respectively, and $\lambda_{c}=c / f_{c}$ the center operational wavelength (with $c$ being the corresponding speed of light). Moreover, $\Gamma_{p q}(t)$ denotes the timemodulated reflection coefficient of the generic $(p, q)$ th element, for which we assume a periodic behavior with period $T_{0}$, viz.,

$$
\Gamma_{p q}(t)=\sum_{n=1}^{L} \Gamma_{p q}^{n} U_{p q}^{n}(t), \quad 0<t<T_{0},
$$

where $U_{p q}^{n}(t)$ denotes a periodic rectangular-pulse function, defined in each period as

$$
U_{p q}^{n}(t)= \begin{cases}1, & (n-1) \tau \leq t \leq n \tau \\ 0, & \text { otherwise }\end{cases}
$$

$L$ is the length of the time-coding sequence, and $\Gamma_{p q}^{n}=\exp \left(j \varphi_{p q}^{n}\right)$ is the reflection coefficient (computed at the center frequency) pertaining the $(p, q)$ th coding element within the interval $(n-1) \tau \leq t \leq n \tau$. By expanding in a Fourier series the time-modulated reflection coefficient in equation (2), and substituting it in equation (1), the farfield scattering patterns for the generic $m$ th harmonic frequency $f_{\mathrm{c}}+m f_{0}$ can be expressed as

$$
\begin{aligned}
& F_{m}(\theta, \varphi)=E(\theta, \varphi) \sum_{q=1}^{N} \sum_{p=1}^{M} a_{p q}^{m} \\
& \quad \times \exp \left\{j \frac{2 \pi}{\lambda_{c}} \sin \theta\left[(p-1) d_{x} \cos \varphi+(q-1) d_{y} \sin \varphi\right]\right\},
\end{aligned}
$$

with the equivalent complex amplitudes $a_{p q}^{m}$ given by

$$
a_{p q}^{m}=\sum_{n=1}^{L} \frac{\Gamma_{p q}^{n}}{L} \sin \mathrm{c}\left(\frac{\pi m}{L}\right) \exp \left[-\frac{j \pi m(2 n-1)}{L}\right] .
$$

Interestingly, these equivalent amplitudes are linear combinations (with complex-valued coefficients) of the quantized reflection coefficients, and thus may exhibit some finer dynamics. For instance, considering a 1-bit (binary) coding, it is actually possible to synthesize equivalent amplitudes characterized by four quantized phase states, that is, an equivalent 2-bit coding, and so on (see [25] for details). However, it is apparent from equation (5) that this reasoning does not apply at the center frequency $(m=0)$, at which only the original coding states can be synthesized.

In essence, the design procedure entails the optimization of the space-time coding in equation (2) in order to synthesize the desired equivalent amplitudes in equation (5), so as to perform simultaneous field manipulations in both space and frequency domains, as described by equation (4).

In what follows, we will illustrate several representative examples of application. 

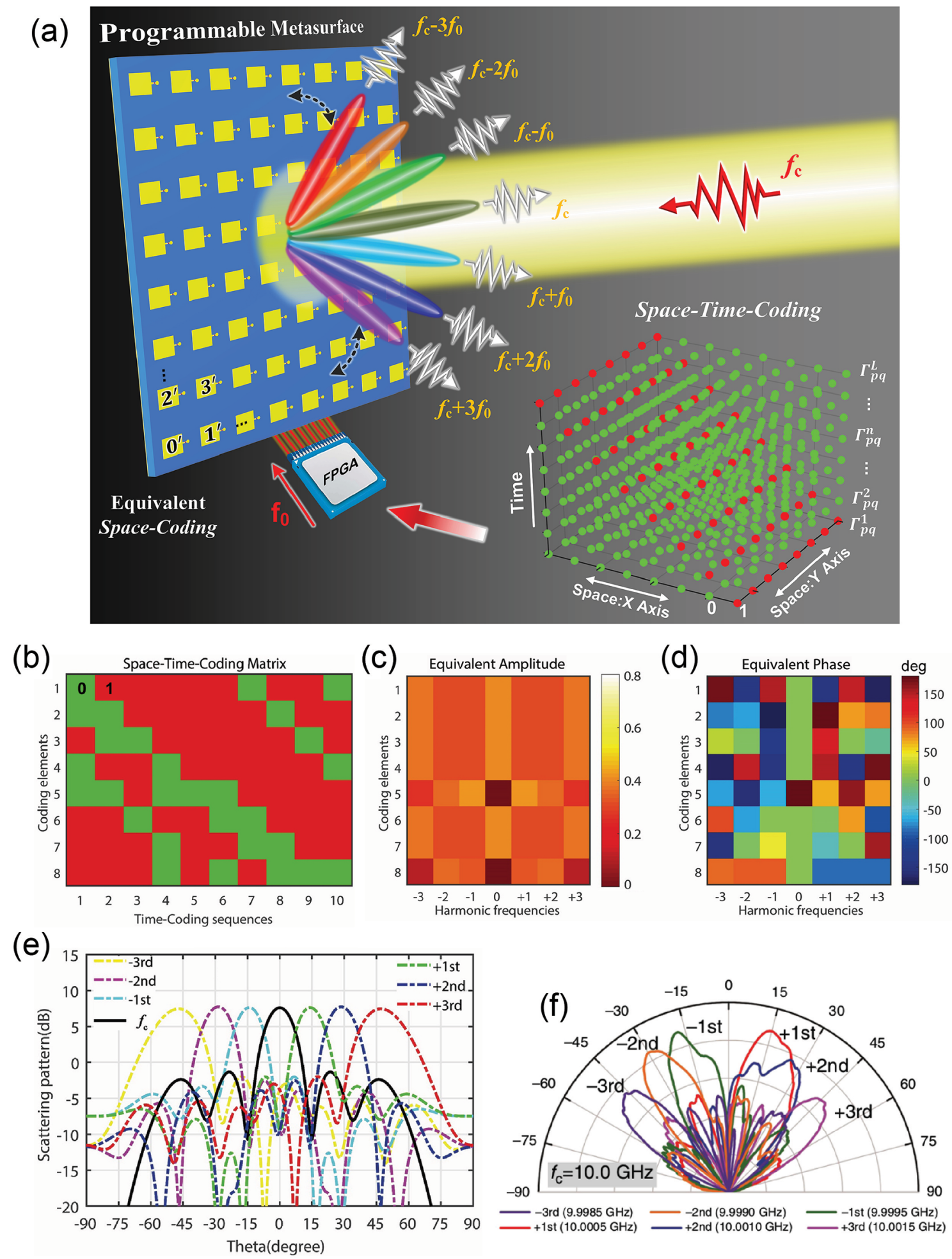

Fig. 1. Harmonic beam steering (adapted from [25]). (a) Conceptual illustration. (b) Optimized space-time-coding matrix. (c, d) Equivalent amplitudes and phase distributions, respectively, at the various harmonic frequencies. (e, f) Simulated and measured, respectively, scattering patterns at various harmonic frequencies. 


\section{Survey of recent advances and applications}

\subsection{Harmonic beam steering/shaping}

An interesting application of the above theory is harmonic beam steering, intended as the deflection of different scattered harmonics toward specific directions in space (see the conceptual illustration in Fig. 1a) [25]. In principle, this can be attained via a simple time-gradient scheme (see, e.g., Figs. $2 \mathrm{a}$ and $2 \mathrm{~b}$ in [25]), which induces different phasegradient distributions in the equivalent amplitudes in equation (5). However, this simple scheme also tends to generate imbalanced amplitude distributions [25]. To overcome this limitation, Figure $1 \mathrm{~b}$ shows a space-time 1-bit coding matrix optimized (via a binary particle-swarm algorithm [40]) so as to equalize the amplitude of the scattered harmonics. In this specific case, an $8 \times 8$-element metasurface is considered, and time-coding sequences have a length of $L=10$. Moreover, since the desired steering effect is along one dimension only, the same time-coding sequence is applied to all elements in each column, so that the 3 -D coding matrix can be effectively flattened to a $2-\mathrm{D}$ form. For an inter-element spacing $d_{x}=d_{y}=\lambda_{c} / 2$, Figures $1 \mathrm{c}$ and $1 \mathrm{~d}$ show the corresponding equivalent amplitude and phase distributions, respectively, from which the amplitude equalization and the appearance of phase gradients at the harmonic frequencies are evident. As anticipated, we note that, in spite of the 1-bit coding utilized, the equivalent phase distributions exhibit a finer (2-bit) quantization, with the exception of the center frequency. This results in the numerically computed scattering patterns are shown in Figure 1e, which display the expected steering effects and equalized amplitudes.

To experimentally validate these results, a microwave prototype operating at a center frequency of $10 \mathrm{GHz}$ was fabricated and characterized. Referring to reference [25] for details, the coding elements consist of metallic patches laid on a grounded dielectric substrate, with an embedded PIN diode to switch between the $1 / 0$ bits (i.e., out of phase responses). Along the lines of the simulated example, the coding elements are grouped in eight columns (each containing eight elements) connected to common biasing lines, and controlled by an FPGA. A modulation frequency $f_{0}=0.5 \mathrm{MHz}$ is assumed, corresponding to a PIN-diode switching speed of $5 \mathrm{MHz}$. Figure 1f shows the measured scattering patterns, which are in fairly good agreement with the simulated results in Figure 1e.

As can be observed from the above example, working with a 1-bit coding does not allow to attain beam steering at the center frequency. As previously mentioned, and better detailed hereafter (see Sect. 3.3), this restriction can be lifted by adopting higher-bit coding schemes (e.g., 2-bit).

In principle, the strategy utilized for steering can also be exploited to realize beam shaping at the harmonic frequencies [25], although there are certain inherent limitations in the joint multi-frequency synthesis (see also the discussion in Sect. 4 below). Other interesting applications include the radar-cross-section reduction/ control by redistributing the scattered power in both space and frequency domains.
Overall, the above results hold interesting promises for a variety of application scenarios, including wireless communications, radars, and holographic imaging.

\subsection{Nonreciprocal reflection effects}

One of the most interesting and promising applications of space-time metastructures is the possibility to break Lorentz reciprocity without the need of magnetic or nonlinear materials. Theoretical [41-43] and experimental [44-46] studies have shown the possibility to attain nonreciprocal reflection or transmission effects via a metasurface imparting a suitable space-time phase gradient. In a recent study [30], this concept was implemented and demonstrated by relying on a space-time coding digital metasurface. Referring to Figure 2a for illustration, an architecture conceptually similar to that in Figure 1a is considered, but now assuming a 2-bit coding, that is, a fourlevel quantization of the phase response (e.g., $0,90^{\circ}, 180^{\circ}$, $270^{\circ}$ ) associated to the digits $0,1,2,3$. Figure $2 \mathrm{~b}$ shows the space-time coding matrix that implements the discretized space-time phase gradient, assuming a $16 \times 16$-element metasurface, and a coding sequence of length $L=4$; also in this case, in view of the 1-D character of the steering effects, the same coding sequence is applied to all elements in each column. As can be observed from the equivalent amplitude and phase distributions shown in Figure $2 \mathrm{c}$ and $2 \mathrm{~d}$, respectively, such space-time coding essentially induces an anomalous reflection, with most of the power concentrated in the $m=1$ harmonic order, which also exhibits an effective phase gradient

$$
\frac{\partial \psi}{\partial x}=-\frac{\pi}{2 d},
$$

with $d=d_{x}=d_{y}$ denoting the constant inter-element spacing. Accordingly, as conceptually illustrated in Figure 2a, assuming a plane wave illumination at frequency $f_{1}=f_{c}$, impinging obliquely at an angle $\theta_{1}$, the dominant scattering contribution will be at a different frequency $f_{2}=f_{c}+f_{0}$ and at an angle [30]

$$
\theta_{2}=\arcsin \left(\frac{\sin \theta_{1}-\frac{\lambda_{\mathrm{c}}}{4 d}}{1+\frac{f_{0}}{f_{c}}}\right) .
$$

If now one considers the time-reversal channel (see Fig. 2a), assuming an incidence at frequency $f_{2}$ and angle $\theta_{2}$, the scattered field does not follow the original forward incidence channel, but it is instead characterized by a different frequency $f_{3}=f_{c}+2 f_{0}$ and a different angle [30]

$$
\theta_{3}=\arcsin \left(\frac{f_{c} \sin \theta_{1}}{f_{c}+2 f_{0}}\right) .
$$

In other words, for oblique incidence $\left(\theta_{1} \neq 0\right)$, the timereversal reflected channel and the forward-incidence one are isolated both spectrally and spatially. It is clear from equations (7) and (8) that the angular separation depends 
(a)

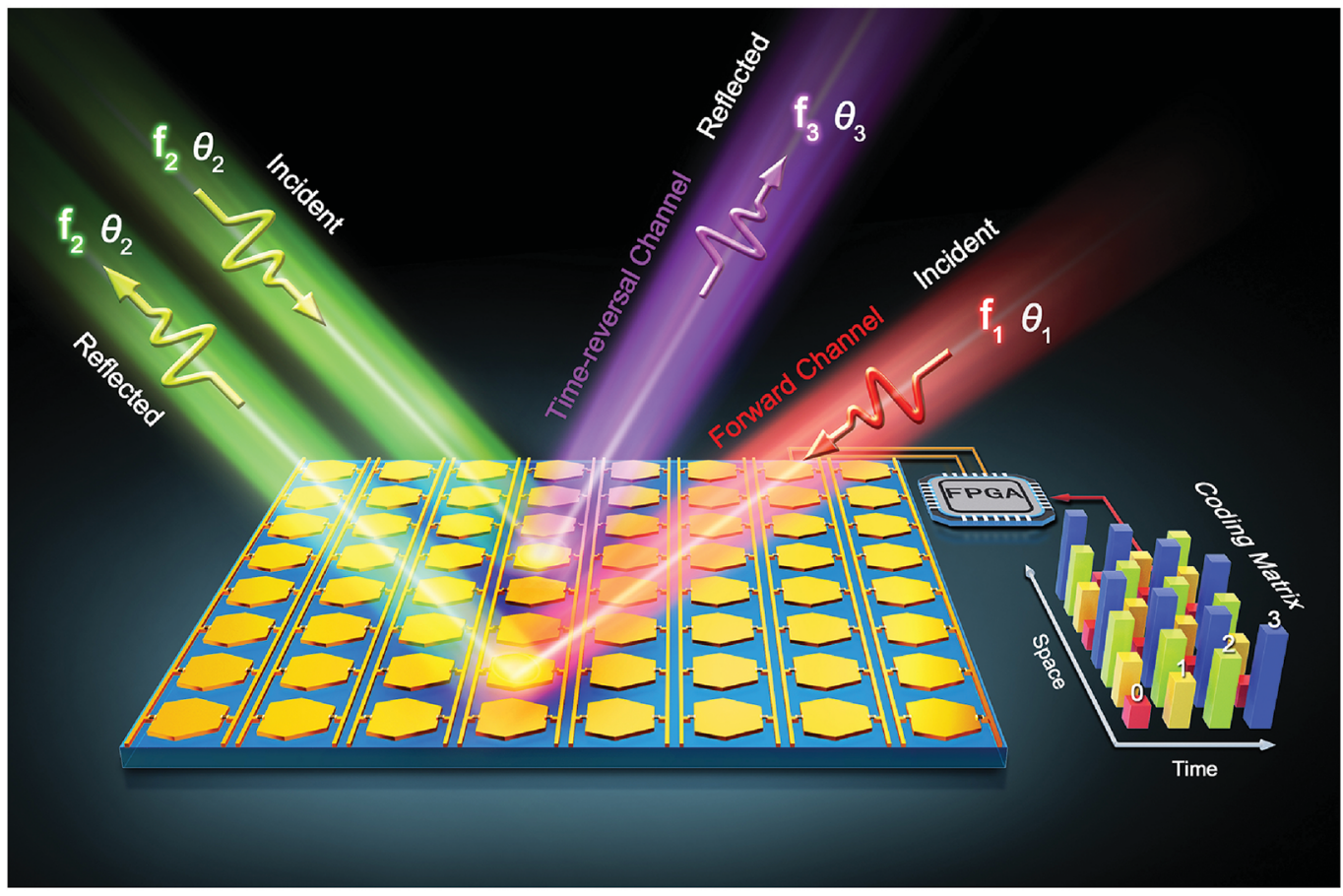

(b)

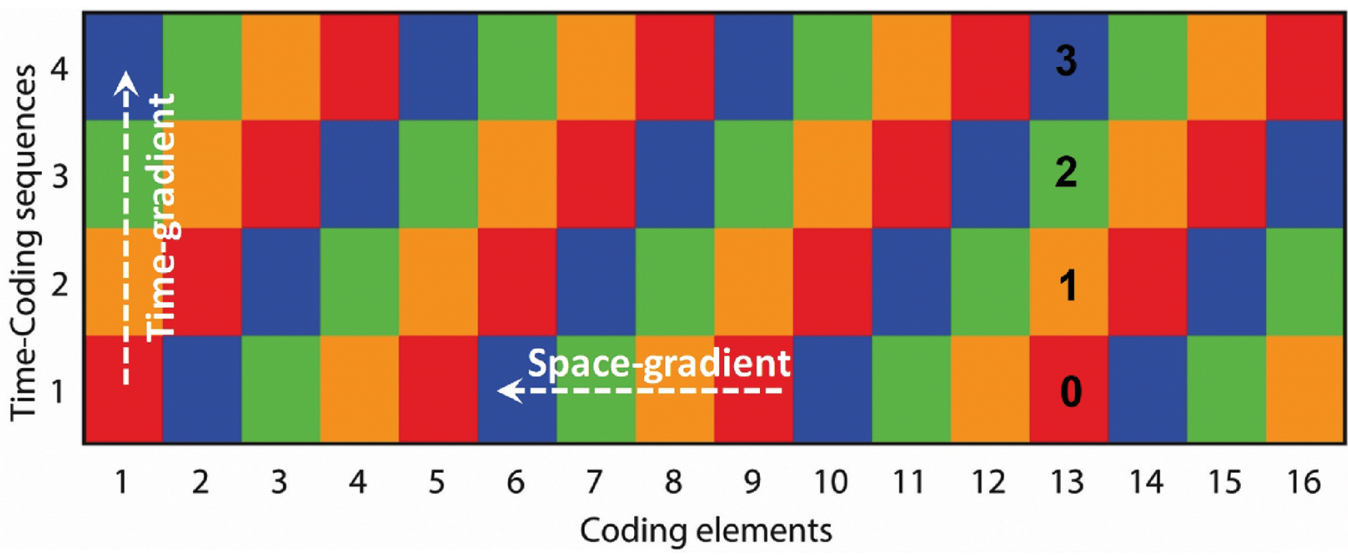

(c)

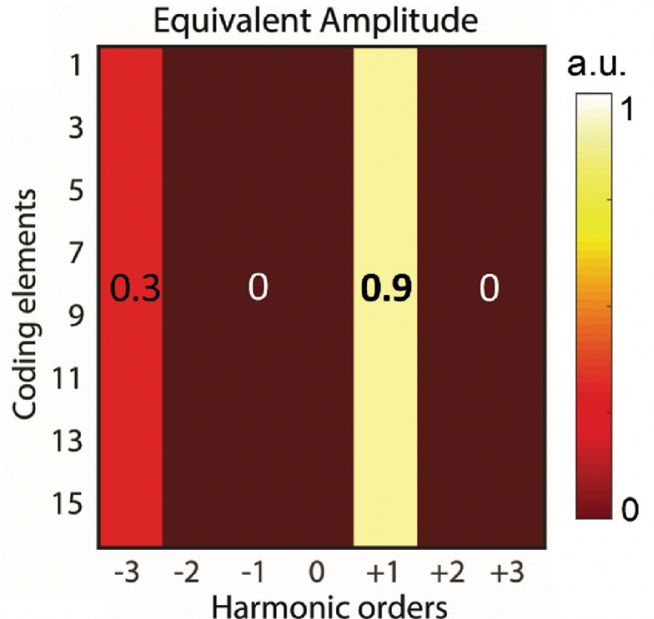

(d)

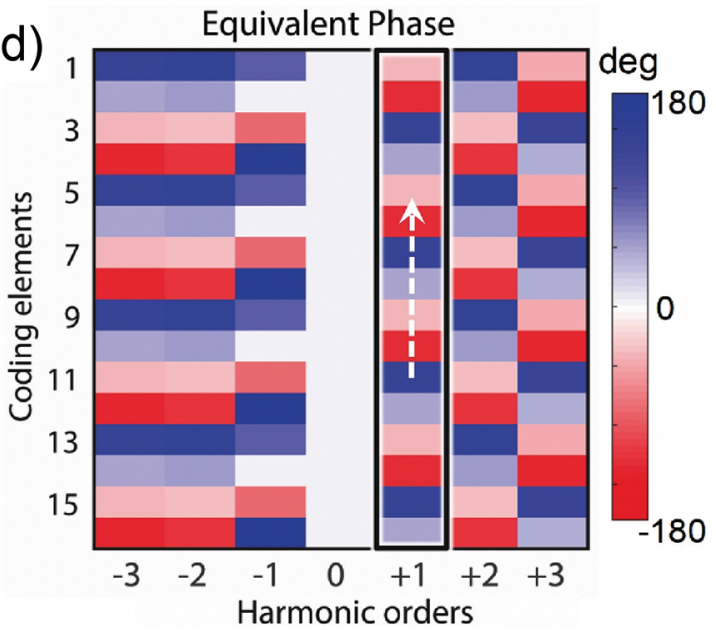

Fig. 2. Nonreciprocal reflection effects (adapted from [30]). (a) Conceptual illustration. (b) Space-time-coding matrix. (c, d) Equivalent amplitudes and phase distributions, respectively, at the various harmonic frequencies. 
(a)

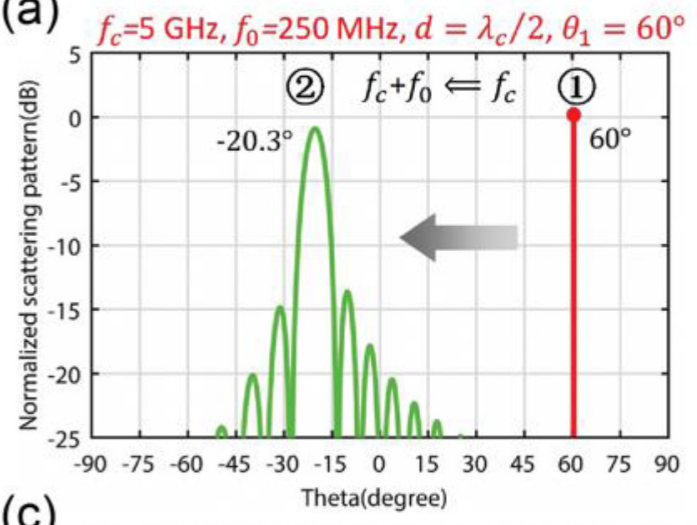

(c)

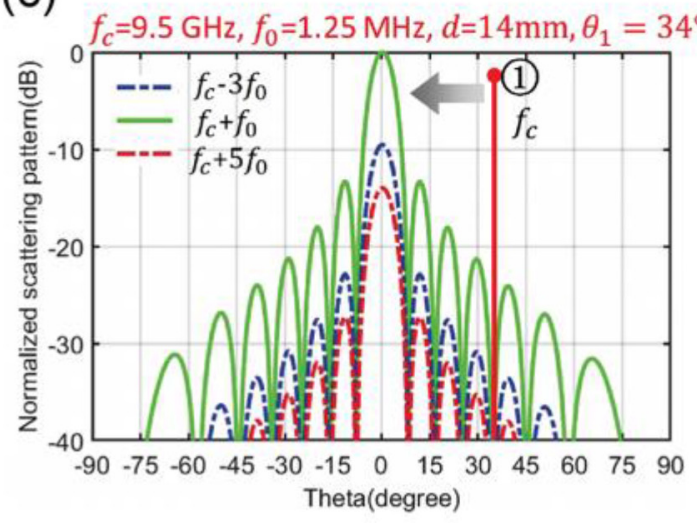

(2) $9.50125 \mathrm{GHz}$

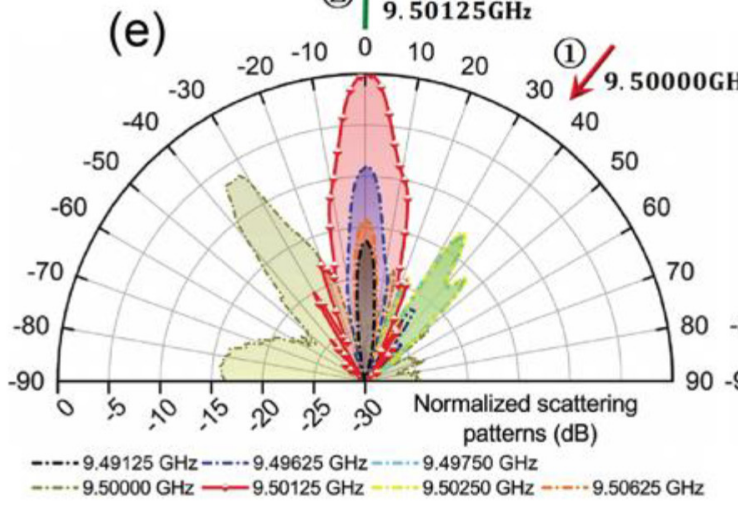

(b)

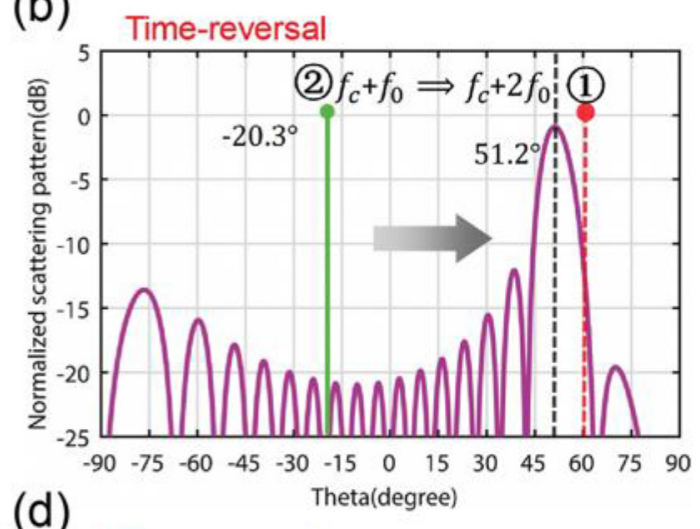

(d)

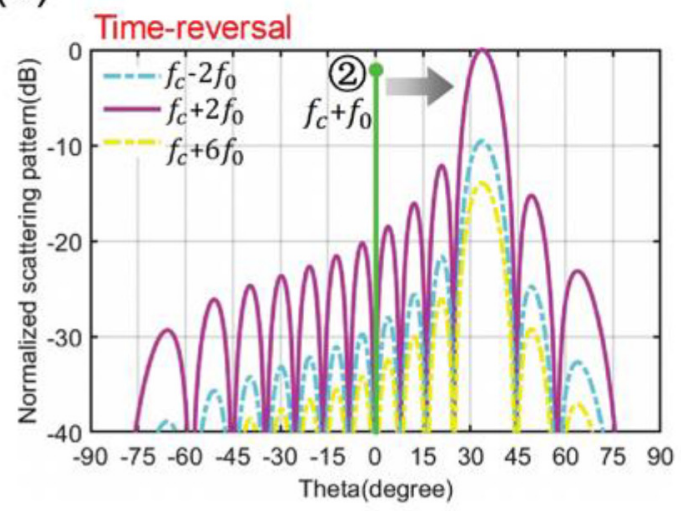

(f)

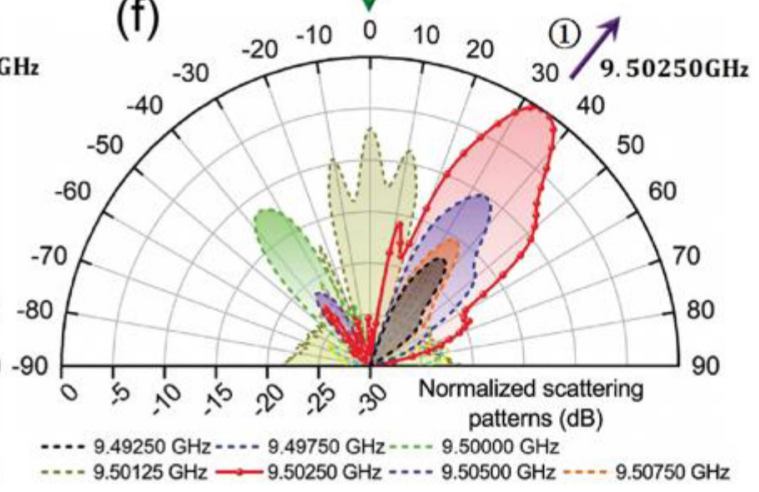

Fig. 3. Nonreciprocal reflection effects (adapted from [30]). (a, b) Numerically computed scattering patterns (forward-incidence and time-reversal channel, respectively) for the dominant harmonic orders, by assuming $f_{c}=5 \mathrm{GHz}, f_{0}=250 \mathrm{MHz}, d=\lambda_{c} / 2$ and $\theta_{1}=60^{\circ}$. $(\mathrm{c}, \mathrm{d})$ Same as above (but including higher-order harmonics) for a more realistic parameter configuration featuring $f_{c}=9.5 \mathrm{GHz}, f_{0}=$ $1.25 \mathrm{MHz}, d=14 \mathrm{~mm}$ and $\theta_{1}=34^{\circ}$. (e, f) Corresponding measured results.

critically on the ratio $f_{0} / f_{c}$. To give an idea, Figures 3 a and $3 \mathrm{~b}$ show some representative results (forward and time-reversal channel, respectively) numerically computed by assuming $f_{c}=5 \mathrm{GHz}, f_{0}=250 \mathrm{MHz}, d=\lambda_{c} / 2$ and $\theta_{1}=60^{\circ}$; in this case, a sizable angular separation of $\sim 9^{\circ}$ is obtained. However, the assumed modulation frequency corresponds to a diode switching frequency of $1 \mathrm{GHz}$, which is not feasible with currently available PIN diodes. Figures $3 \mathrm{c}$ and $3 \mathrm{~d}$ show the results for a more realistic parameter configuration $\left(f c=9.5 \mathrm{GHz}, f_{0}=1.25 \mathrm{GHz}\right.$, that is, a diode switching speed of $5 \mathrm{MHz}$ ), for which the angular separation is $<1^{\circ}$. Although such angular separation could not be resolved with the available measurement setup, it was still possible to validate experimentally the frequency separation. To this aim, a microwave prototype with coding elements embedding two PIN diodes was fabricated, so as to attain the required four quantized phase responses (see [30] for details). Figures 3e and 3f show the measured scattering patterns pertaining to the forward-incidence and time-reversal channel, respectively, from which a good agreement with the theoretical predictions can be observed. 

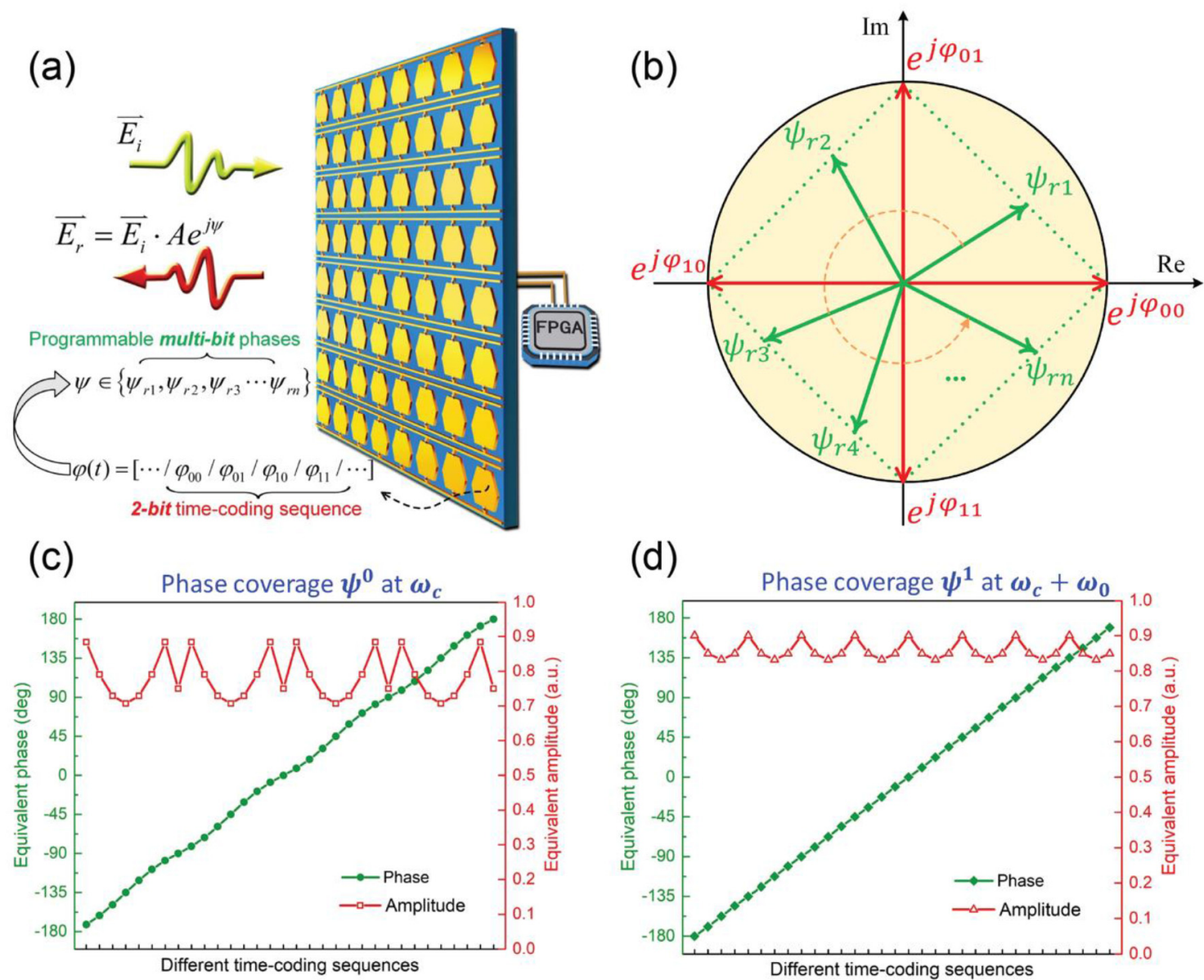

Fig. 4. Arbitrary multi-bit programmable phases (adapted from [29]). (a) Conceptual illustration. (b) Basic illustration of the vector synthesis method in the complex plane for the generation/analysis of multi-bit phases. (c, d) Equivalent phase coverage at the center angular frequency $\omega_{c}=2 \pi f_{c}$ and the first harmonic $\omega_{c}+\omega_{0}=2 \pi\left(f_{c}+f_{0}\right)$, respectively, for a 2-bit time-coding sequences with length $L=8$.

The above results may found interesting applications to wireless communications and radar systems, for separating the transmitting and receiving channels. It is also worth highlighting that, thanks to the inherently programmable character of the architecture, nonreciprocal effects can also be realized "on-demand."

\subsection{Arbitrary multi-bit programmable phases}

As previously mentioned, higher-bit programmable metasurfaces exhibit lower quantization phase error and therefore enable a more precise control of EM waves. For instance, in reference [25], it was shown that a 2-bit coding could be effectively exploited to realize beam steering and beam shaping at the center frequency. So far, 1-bit and 2-bit programmable metasurfaces have been successfully realized, but it is very challenging to attain higher-bit programmable phases via PIN-diode-based metasurfaces. Alternative switching strategies (based, e.g., on varactors, MEMS, graphene, liquid crystals) also exhibit limited phase coverage and cannot realize continuous $360^{\circ}$ phase modulation.
Recently, in [29], a 2-bit programmable metasurface combined with a time-coding approach was proposed, which can attain arbitrary multi-bit and even quasicontinuous programmable phases, as schematically illustrated in Figure 4a. Specifically, a method of vector synthesis (illustrated in Fig. 4b) was put forward to analyze and synthesize the phase coverages. For instance, 4-bit and arbitrarily higher-bit phases can be synthesized via a 2-bit coding metasurface, simply by manipulating the timecoding sequences. Several numerical examples of beam steering, at both the center and harmonic frequencies, were illustrated to demonstrate the effectiveness of the proposed approach. Figures $4 \mathrm{c}$ and $4 \mathrm{~d}$ show that the phase coverage at the center frequency and the first harmonic frequency can reach $360^{\circ}$ with high amplitudes by suitably designing the 2-bit time-coding sequences. For experimental validation, an FPGA-controlled prototype working at microwave frequencies was fabricated and experimentally characterized (see [29] for details). Overall, this approach provides a simple way to design arbitrary multi-bit programmable metasurfaces without the requirement of complicated structural design and control systems. This may find 


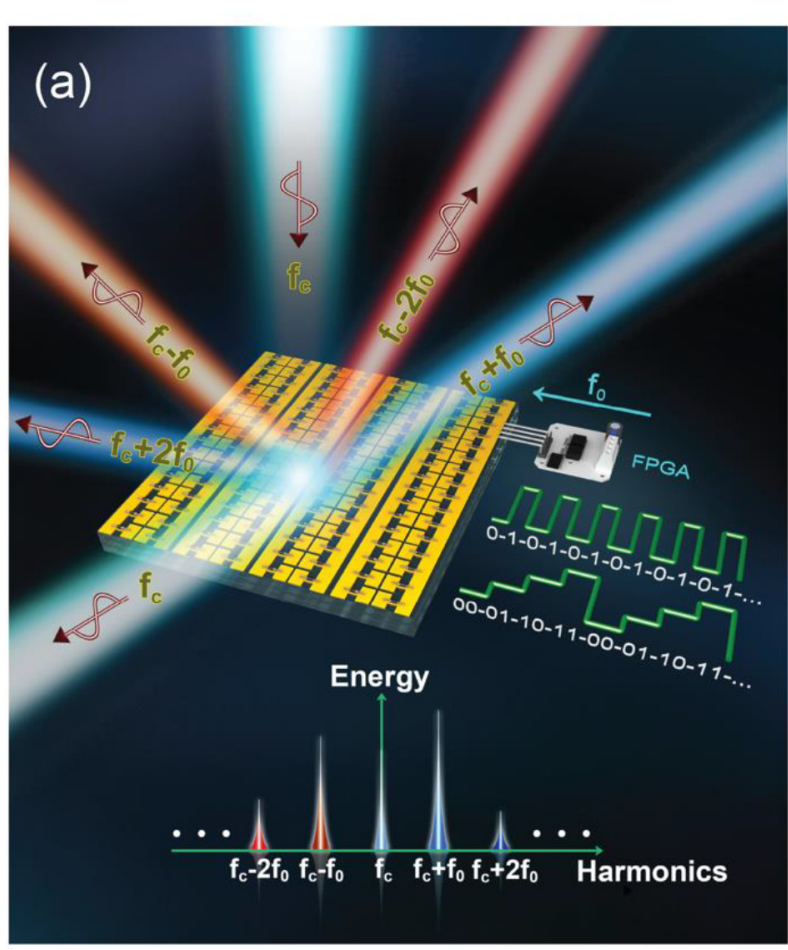

(b)
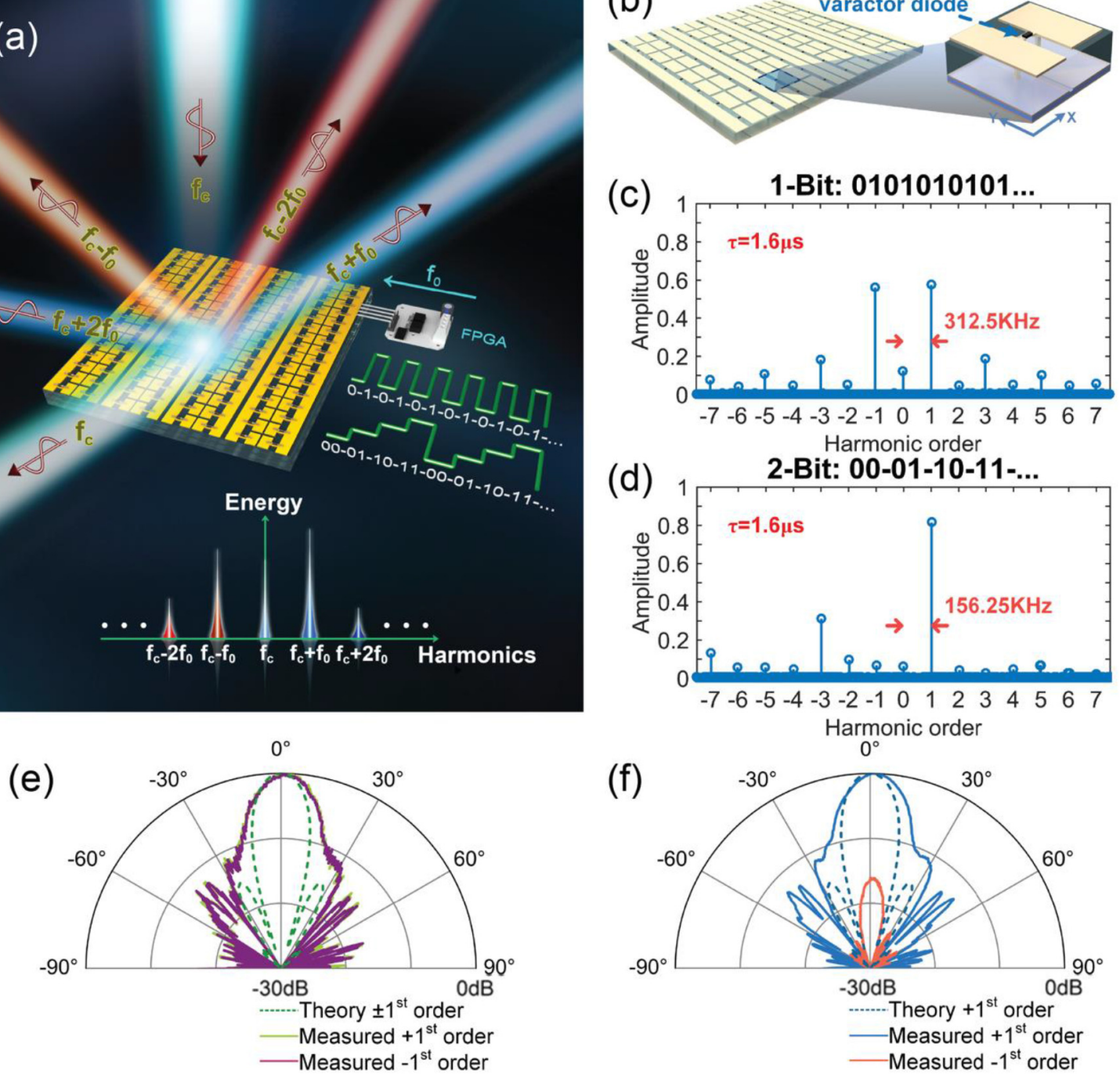

Fig. 5. Nonlinear harmonic manipulation via time coding (adapted from [32]). (a) Conceptual illustration. (b) Schematic of a coding element featuring a varactor diode. (c, c) Examples of measured spectral distributions, for 1-bit and 2-bit coding, respectively. (e, f) Corresponding scattering patterns (theoretical and measured) for the harmonic orders +1 and -1 .

important applications to high-performance antennas, high-resolution imaging, as well as high-capacity wireless communications and radar systems.

\subsection{Non-linear harmonic manipulations via time coding}

Other interesting time-varying architectures can be implemented via the use of varactor (instead of PIN) diodes, which enables more flexibility in the modulation waveform. For instance, in reference [32], a time coding metasurface was proposed that possesses powerful capabilities in manipulating the spectral distribution of EM fields, as conceptually illustrated in Figure 5a. In this prototype study, only temporal coding was considered, assuming a space-invariant temporal modulation of the reflection coefficient according to a periodic function $\Gamma(t)$, with period $T_{0}$. Accordingly, assuming time-harmonic plane-wave illumination at frequency $f_{c}$, the general theory in equation (1) can be particularized to express the spectrum of the reflected wave $E_{r}$ in terms of the incident one $E_{i}$ as follows [32],

$$
\begin{aligned}
E_{r}(f) & =E_{i}(f) * \Gamma(f)=\delta\left(f-f_{c}\right) * \sum_{k=-\infty}^{\infty} a_{k} \delta\left(f-k f_{0}\right) \\
& =\sum_{k=-\infty}^{\infty} a_{k} \delta\left(f-f_{c}-k f_{0}\right),
\end{aligned}
$$

where $\delta(\cdot)$ denotes the Dirac delta function, $*$ is the convolution operation, and $a_{k}$ represents the complex Fourier coefficient of the $k$ th-order harmonic. As previously mentioned, although the reflection coefficient is defined for 
a linear time-invariant system, equation (9) is approximately valid in the adiabatic limit $f_{0} \ll f_{c}[38,39]$. From equation (9), it is possible, in principle, to attain arbitrary harmonic distributions by judiciously designing the temporal modulation for the reflection coefficient $\Gamma(t)$, which opens up interesting pathways in the spectral shaping of EM waves.

Figure 5b illustrates a practical implementation of a coding element, featuring two rectangular patches linked by a varactor diode acting as a tunable element, controlled via a biasing voltage [32]. Accordingly, narrow slots are etched on the bottom plate so as to bias the diodes through metallic vias. Also in this architecture, an FPGA-based control platform is exploited to generate the required driving signals so as to implement different functionalities in the reflected-wave spectrum.

Figures 5c-5f illustrate some representative experimental results obtained by means of a microwave prototype (see [32] for details). Specifically, Figure 5c shows the measured spectral distributions pertaining to a binary phase modulation associated to the time-domain digital coding $01010101 \ldots$, with the " 0 " and " 1 " bits denoting $0^{\circ}$ and $180^{\circ}$ phase levels, respectively (corresponding to biasing voltages of 0 and $-9 \mathrm{~V})$. Consistently with the theoretical predictions from equation (9), most of the impinging power (at the center frequency) is transferred to higher-order harmonics (most notably, the orders +1 and -1 ); the small residual at the center frequency is attributable to modeling approximations and uncertainties, as well as fabrication tolerances. As can be observed from the measured scattering patterns in Figure 5e, the harmonic orders +1 and -1 exhibit similar shapes and comparable amplitudes. Figure 5d shows instead the spectral distribution pertaining to a 2 -bit coding, with four digital states " $00 "$, " 01 ", "10" and " 11 " corresponding to reflection phases $0^{\circ}, 90^{\circ}, 180^{\circ}$ and $270^{\circ}$, respectively (obtained via biasing voltages $0,-6,-9$ and $-21 \mathrm{~V})$. In this case, due to the time-gradient enforced, the spectral symmetry is broken and most of the power is transferred to the harmonic order +1 . As apparent from the scattering patterns shown in Figure 5f, the harmonic orders +1 and -1 maintain similar shapes, but now the amplitudes are significantly different.

The above examples illustrate the potential of the proposed approach in controlling the spectral distribution of the reflected EM waves. This concept may find important applications to the development of simplified and compact communication and radar systems, and can be extended from the microwave range to the optical regime and, in principle, to acoustic scenarios.

\subsection{Independent control of harmonic amplitudes and phases}

A major limitation of space-time coding digital metasurfaces stems from the inherent coupling effects between the amplitude and phase responses at the various harmonic orders, as can be understood from the expression of the equivalent complex-valued amplitudes in equation (5). In [31], an analytical method was proposed for independent control of the harmonic amplitudes and phases via spacetime coding. Referring to the conceptual illustration in
Figure 6a, the reflection phase of the coding element is temporally modulated with a periodic square wave between two selected phase states $\phi_{1}$ and $\phi_{2}$; moreover, for a more flexible control, a time delay $t_{0}$ is introduced in the reflection coefficient. For simplicity, no spatial coding is assumed for now. Accordingly, the reflected-wave spectrum in equation (9) can be rewritten as

$$
\begin{aligned}
E_{r}(f)= & 2 \pi A \cos \left(\frac{\phi_{1}-\phi_{2}}{2}\right) \exp \left[j\left(\frac{\phi_{1}+\phi_{2}}{2}\right)\right] E_{i}(f) \\
& +\sum_{m=-\infty}^{\infty}\left(\frac{4 A}{2 m-1}\right) \sin \left(\frac{\phi_{1}-\phi_{2}}{2}\right) \\
& \times \exp \left\{j\left[\frac{\phi_{1}+\phi_{2}}{2}-(2 m-1) 2 \pi f t_{0}\right]\right\} \\
& \times E_{i}\left[f-(2 m-1) f_{0}\right],
\end{aligned}
$$

with $A$ denoting a constant reflection amplitude. From equation (10), we notice that the amplitude of the harmonics can be tuned by acting on the phase difference $\left(\phi_{1}-\phi_{2}\right)$. Moreover, the time delay $t_{0}$ offers an additional degree of freedom to tailor the phase response, while maintaining the amplitude unchanged, thereby enabling independent control. In addition, since the same time delay yields different phase lags at various harmonics, it can also be exploited to realize simultaneous multi-order harmonic manipulations.

Figure $6 \mathrm{~b}$ illustrates the geometry of a coding element, featuring rectangular patches loaded via four varactor diodes; by suitably changing the biasing voltages, the phase response can be tuned over a broad range $\left(\sim 270^{\circ}\right)$ [31]; once again, an FPGA controller is utilized to generate the required coding signals. A prototype working at microwave frequencies was fabricated and characterized for experimental validation. Referring to reference [31] for details, Figure 6c shows the measured amplitudes and phases at various harmonic orders, for three different combinations of the biasing voltages (applied to all coding elements); for simplicity, the varactors in each column share the same biasing voltage, and therefore there are only two control voltages. As can be observed, it is possible to manipulate the amplitude and phase responses in the spectrum by varying the time coding. Next, spatial coding is introduced by independently modulating the columns of the metasurface elements, thereby enabling some beam-shaping capabilities. For illustration, Figure $6 \mathrm{~d}$ shows three representative patterns measured at the harmonic order +1 , corresponding to different spatial codings. As can be observed, by acting on the time coding, it is possible to control the scattering amplitude, while maintaining the beam shape. Finally, as illustrated in Figure 6e, by introducing and controlling a time delay $t_{0}$ in the reflection coefficient, it is possible to tailor the scattering patterns at different harmonic orders, in good agreement with the theoretical predictions [31].

The above results open up interesting possibilities for harmonic manipulations, with potential applications ranging from wireless communications to radars. 
(a)
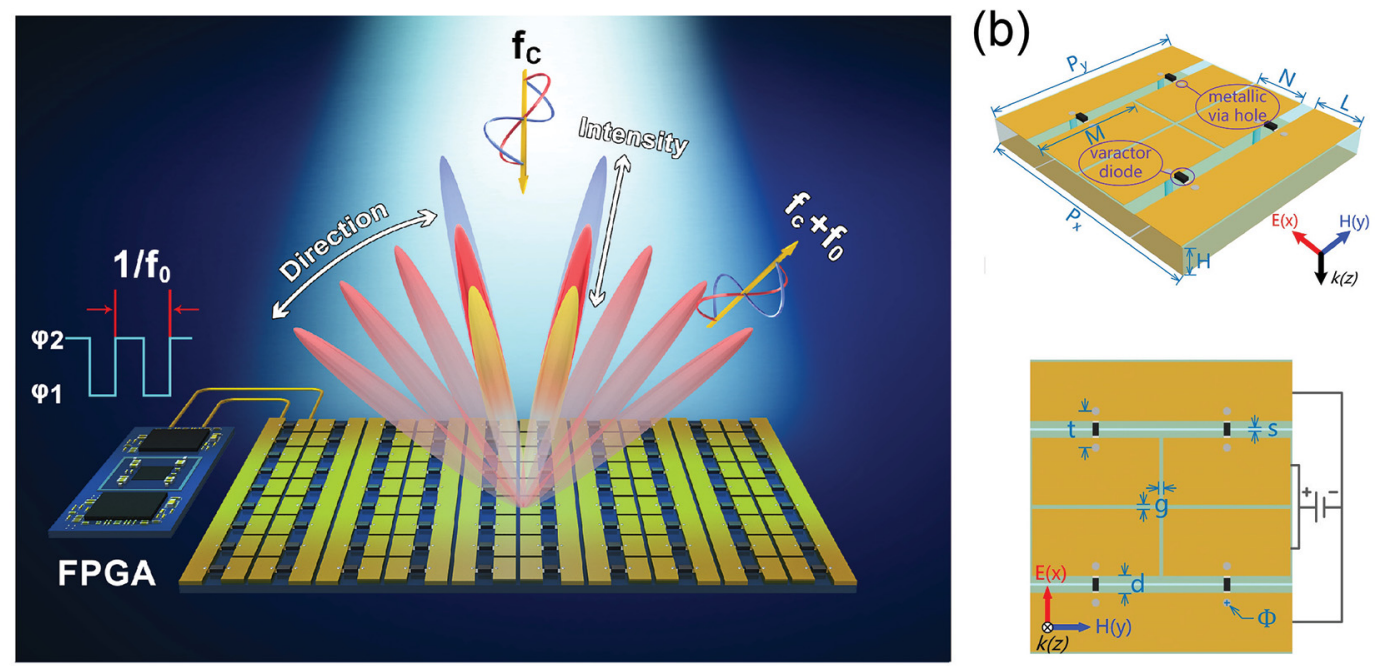

(c)

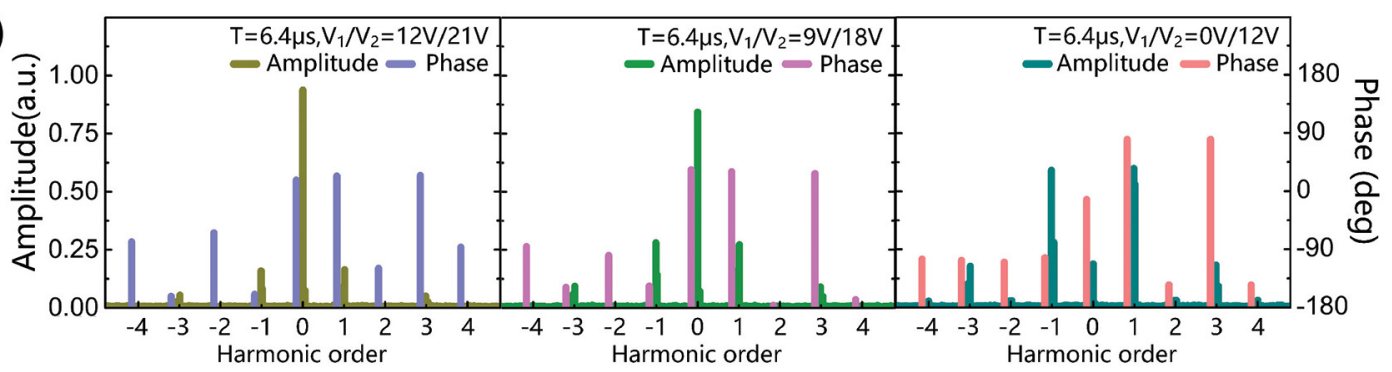

(d)

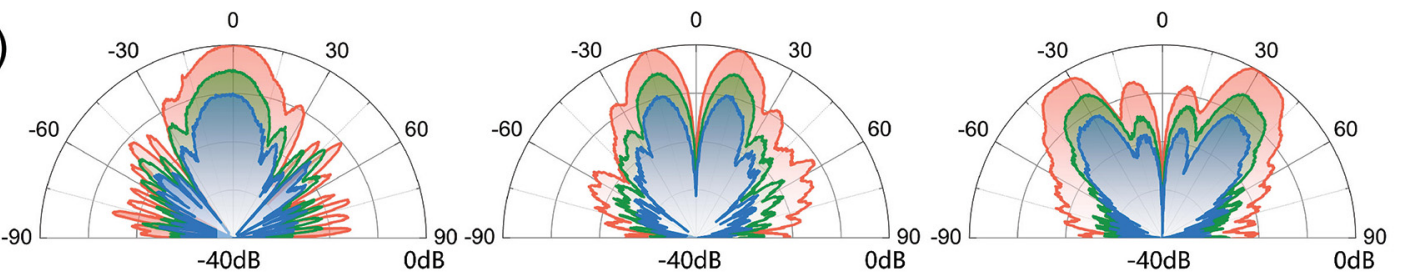

(e)
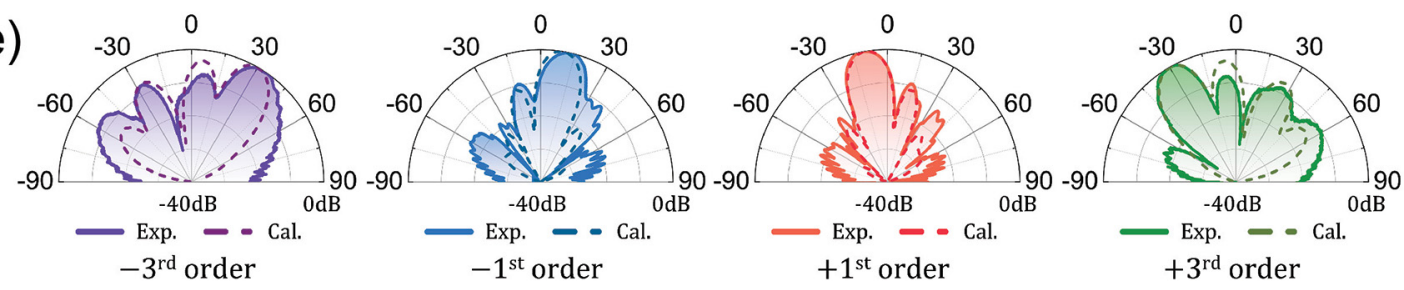

Fig. 6. Independent control of harmonic amplitudes and phases (adapted from [31]). (a) Conceptual illustration. (b) Schematic of the coding element featuring four varactor diodes. (c) Measured amplitudes and phases at various harmonic orders, for different combinations of the biasing voltages. (d) Measured scattering patterns at the harmonic order +1 , for three different spatial codings; patterns of different colors illustrate the possibility to independently control the amplitude by acting on the temporal coding. (e) Representative examples of multi-order harmonic tailoring of the scattering patterns (measured and theoretical).

\subsection{Applications to wireless communications}

The capabilities enabled by space-time coding digital metasurfaces in terms of manipulating the spatial and spectral distributions of EM waves can be exploited to directly embed digital information in microwave carrier signals, without the need of conventional radio frequency circuits. This sets the stage for novel architectures of wireless communication systems [32,35,36,47-49].
For instance, we observe from Figures $5 \mathrm{c}$ and $5 \mathrm{~d}$ that the power can be efficiently transferred from the center frequency to the harmonic orders +1 and -1 via suitably designed temporal codings. One may interpret the incident wave and the time-varying reflection coefficient as the carrier wave and baseband signal, respectively, of traditional communication systems. Accordingly, direct modulation can be attained by establishing a suitable mapping between the baseband information and the reflection coefficient. 


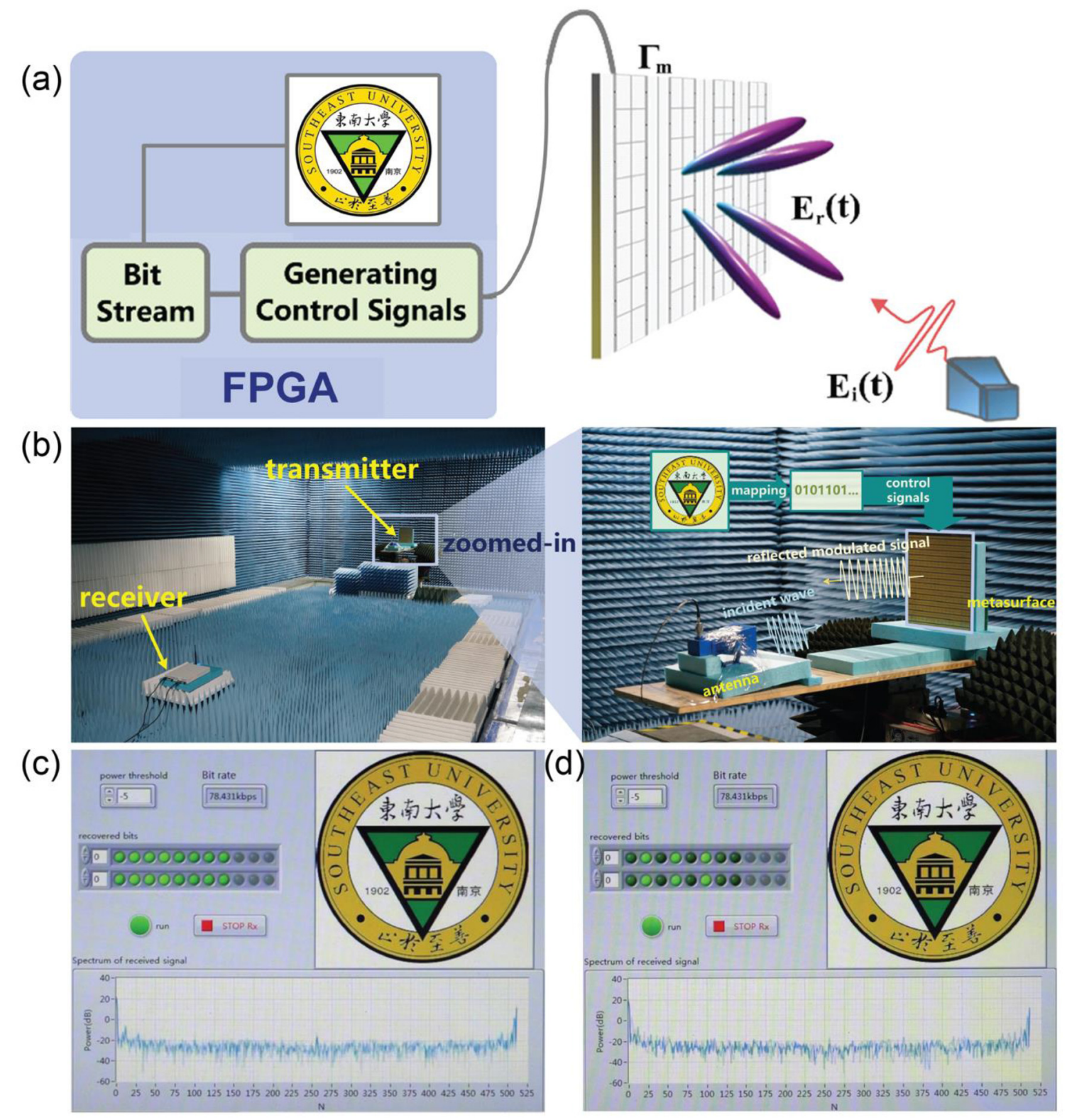

Fig. 7. BFSK transmission system based on space-time coding digital metasurfaces (adapted from [32]). (a) Conceptual illustration. (b) Photographs of the prototype and measurement setup. (c, d) Measurement results with receiving angle of $0^{\circ}$ and $30^{\circ}$, respectively.

Figure 7a shows the schematic of a transmitter based on a time coding metasurface, which was proposed in [32]. In this case, a binary frequency shift keying (BFSK) modulation scheme is implemented, which exploits a pair of discrete frequencies to represent the binary digits " 0 " and "1". As a first step, the FPGA controller generates a bit stream of the information that needs to be transmitted (a picture, in the schematic). Subsequently, the bit streams are mapped onto corresponding time coding sequences, which are repeated periodically so as to produce a pair of discrete frequencies according to the BFSK scheme. In this way, the digital information is directly embedded in the reflected EM wave, and hence transmitted. Figure $7 \mathrm{~b}$ shows a microwave prototype that was fabricated and tested for experimental validation (see [32] for details). Figures $7 \mathrm{c}$ and $7 \mathrm{~d}$ show some representative measurements results, demonstrating the possibility to transmit and correctly receive a high resolution picture.

Another interesting example was proposed in reference [35], implementing the quadrature phase shift keying (QPSK) modulation scheme. Referring to [35] for details, the modulated reflection coefficient can be written as the superposition of scaled and shifted pulses

$$
\begin{gathered}
\Gamma(t)=\Gamma_{m} g(t), \quad 0 \leq t \leq T \\
\Gamma_{m} \in M=\left\{\exp \left(-j \frac{3 \pi}{4}\right), \exp \left(-j \frac{\pi}{4}\right), \exp \left(j \frac{\pi}{4}\right), \exp \left(j \frac{3 \pi}{4}\right)\right\} \\
|M|=4, m=0,1,2,3
\end{gathered}
$$




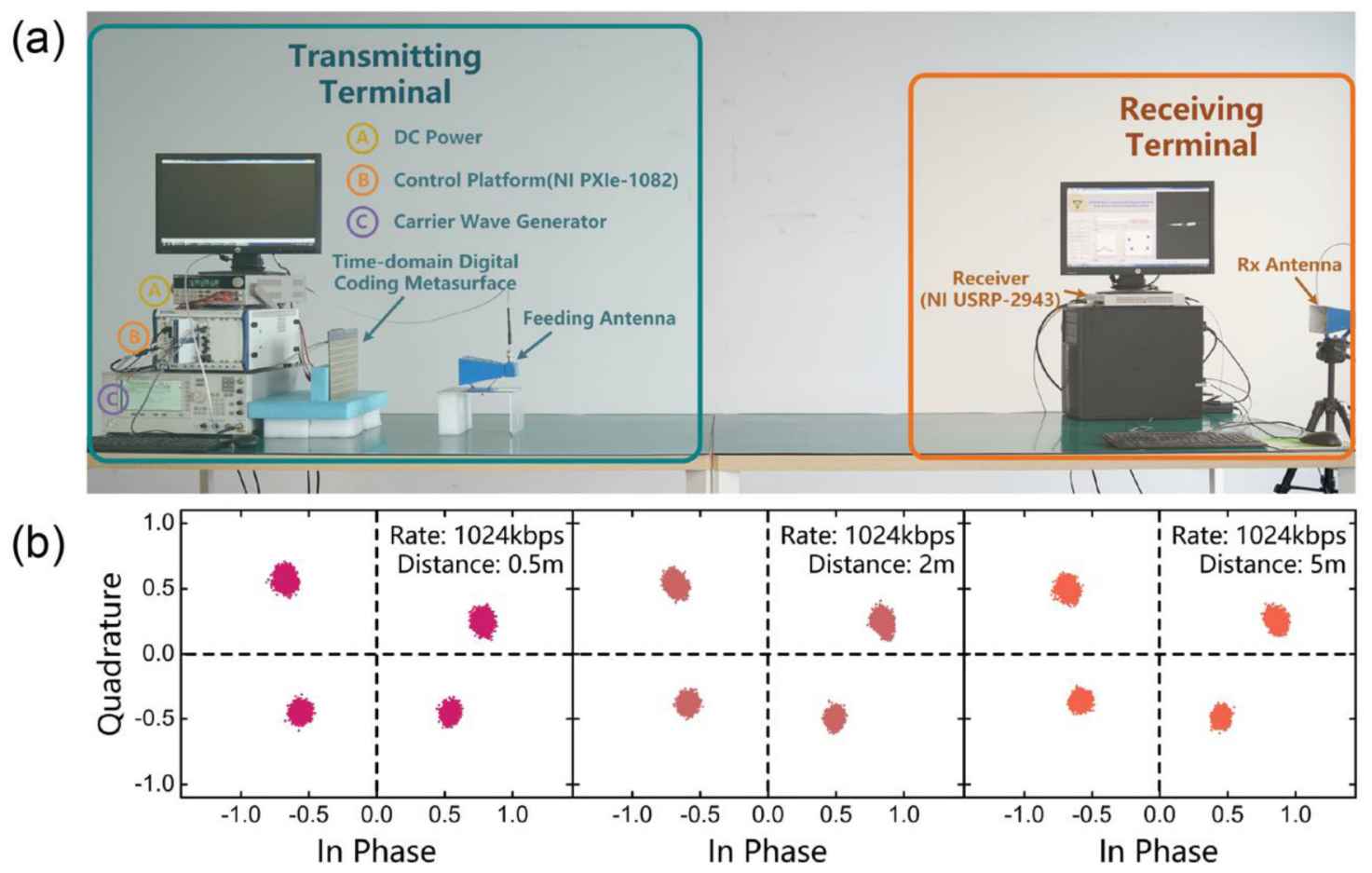

Fig. 8. QPSK transmission system based on space-time coding digital metasurfaces (adapted from [35]). (a) Photograph of prototype and measurement setup in an indoor scenario. (b) Received constellation diagrams for different transmission distances.

where $g(t)$ represents a basic pulse shaping function, $T$ is the period of message symbol, $M$ is a set of constellation points with cardinality $|M|=4$, and $\Gamma_{m}$ is the reflection coefficient during one message symbol. The constellation points in $M$ are associated to 2-bit digital states " 00 ", " 10 ", "11", and "01", respectively. By comparison with the previous BFSK system, the QPSK system is more efficient in the information modulation due to the shorter length of the time coding sequence required during each message symbol, and can therefore reach higher transmission rates.

Also in this case, a proof-of-concept validation was carried out via a microwave prototype (see [35] for details). Figure 8a shows a photograph of the realized QPSK system for data transmission in an indoor scenario. Figure $8 \mathrm{~b}$ shows the corresponding received constellation diagrams, for different transmission distances. Real-time video transmission was demonstrated with a high data rate of about $1.6 \mathrm{Mbps}$.

Overall, the above results confirm the practical feasibility of wireless communication systems based on time-coding metasurfaces, which offer significant simplifications in the architecture and reduced power consumption, without sacrificing the system performance.

\section{Conclusions and outlook}

In summary, digital programmable metasurfaces constitute a very powerful and versatile platform for implementing spatio-temporal modulation schemes, which significantly expand and generalize concepts such as time-modulated arrays and phase-switched screens. In particular, the temporal dimensionality represents a crucial addition within the emerging paradigm of "information metastructures" [19,20], enabling advanced (possibly non-reciprocal) field-manipulation capabilities jointly in the spectral and spatial domains. In this paper, we have reviewed some recent advances and applications, including, harmonic beam steering/shaping, nonreciprocal effects, harmonic manipulations, and wireless communications.

Among the most interesting perspectives for these concepts it is worth mentioning the field of "intelligent reflecting surfaces", which is establishing itself as a pillar for future wireless communication networks [49-53]. Within this framework, space-time coding digital metasurfaces can effectively replace the required decoding, encoding, and radio-frequency processing blocks, also enabling softwaredefined, self-adaptive, and cognitive functionalities. Further flexibility may be endowed by transmission-type and transmission-reflection-integrated platforms, that are currently being explored, together with hybrid (phase/ amplitude) modulation schemes.

Other interesting and promising application scenarios include next-generation radars, scattering-signature reduction/control, imaging and processing systems. Also of great interest are applications to terahertz frequencies and other physical domains (e.g., acoustics).

As previously mentioned, one limitation of the basic harmonic beam steering/shaping scheme presented in Section 3.1 is the inherently entangled character of multifrequency syntheses. This stems from the fact that single 
coding-element response generally affects all harmonic orders, as apparent from the equivalent amplitudes in equation (5). We have shown (see Sect. 3.5) a possible strategy for independent control of amplitude and phase, which enables some multi-order harmonic manipulations. We are currently working on the implementation of more advanced space-time coding schemes [54], whereby the scattering patterns at different harmonics can be synthesized simultaneously and independently. These schemes essentially rely on more sophisticated temporal coding sequences, which exploit digital-filtering concepts to separately control the scattering responses at the various harmonic orders. Preliminary numerical and experimental results look encouraging.

Another critical aspect is related to the modulation frequency, which is inherently limited by the switching speed of the diode element. For instance, in the nonreciprocal-reflection scenario illustrated in Section 3.2, the reliance on PIN diodes severely curtailed the angular separation between the forward-incidence and time-reversal channels, thereby preventing a complete experimental observation of the phenomenon (see Figs. 3e and 3f). It appears therefore crucial to implement faster switching schemes, also with a view toward applications to higher frequencies (e.g., terahertz). Within this framework, emerging platforms based on graphene [55-57] and vanadium dioxide $[58,59]$ seem very promising [60].

\section{References}

1. C. Della Giovampaola, N. Engheta, Nat. Mater. 13, 1115 (2014)

2. T.J. Cui, M.Q. Qi, X. Wan, J. Zhao, Q. Cheng, Light. Sci. Appl. 3, e218 (2014)

3. R.G. Parker, R.L. Rardin, Discrete optimization (Academic Press, San Diego, CA, 2014)

4. M. Paquay, J.-C. Iriarte, I. Ederra, R. Gonzalo, P. de Maagt, IEEE Trans. Antennas Propagat. 55, 3630 (2007)

5. H. Yang, X. Cao, F. Yang, J. Gao, S. Xu, M. Li, X. Chen, Y. Zhao, Y. Zheng, S. Li, Sci. Rep. 6, 35692 (2016)

6. C. Huang, B. Sun, W. Pan, J. Cui, X. Wu, X. Luo, Sci. Rep. 7, $42302(2017)$

7. L. Li, T.J. Cui, W. Ji, S. Liu, J. Ding, X. Wan, Y.B. Li, M. Jiang, C.-W. Qiu, S. Zhang, Nat. Commun. 8, 197 (2017)

8. L. Li, H. Ruan, C. Liu, Y. Li, Y. Shuang, A. Alù, C.-W. Qiu, T.J. Cui, Nat. Commun. 10, 1082 (2019)

9. R.Y. Wu, L. Bao, L.W. Wu, Z.X. Wang, Q. Ma, J.W. Wu, G. D. Bai, V. Galdi, T.J. Cui, Adv. Optical Mater. 8, 1902126 (2020)

10. L. Zhang, X. Wan, S. Liu, J.Y. Yin, Q. Zhang, H.T. Wu, T.J. Cui, IEEE Trans. Antennas Propagat. 65, 3374 (2017)

11. M. Moccia, S. Liu, R.Y. Wu, G. Castaldi, A. Andreone, T.J. Cui, V. Galdi, Adv. Opt. Mater. 5, 1700455 (2017)

12. T.J. Cui, S. Liu, G.D. Bai, Q. Ma, Research 2019, 2584509 (2019)

13. S. Liu, T.J. Cui, L. Zhang, Q. Xu, Q. Wang, X. Wan, J.Q. Gu, W.X. Tang, M. Qing Qi, J.G. Han, W.L. Zhang, X.Y. Zhou, Q. Cheng, Adv. Sci. 3, 1600156 (2016)

14. S. Liu, A. Noor, L.L. Du, L. Zhang, Q. Xu, K. Luan, T.Q. Wang, Z. Tian, W.X. Tang, J.G. Han, W.L. Zhang, X.Y. Zhou, Q. Cheng, T.J. Cui, ACS Photonics 3, 1968 (2016)
15. L. Zhang, R.Y. Wu, G.D. Bai, H.T. Wu, Q. Ma, X.Q. Chen, T.J. Cui, Adv. Funct. Mater. 11, 1802205 (2018)

16. B. Xie, K. Tang, H. Cheng, Z. Liu, S. Chen, J. Tian, Adv. Mater. 29, 1603507 (2017)

17. T.J. Cui, S. Liu, L.L. Li, Light Sci. Appl. 5, e16172 (2016)

18. H. Wu, G.D. Bai, S. Liu, L. Li, X. Wan, Q. Cheng, T.J. Cui, Natl. Sci. Rev. 7, 561 (2020)

19. L. Li, T.J. Cui, Nanophotonics 8, 703 (2019)

20. T.J. Cui, L. Li, S. Liu, Q. Ma, L. Zhang, X. Wan, W.X. Jiang, Q. Cheng, iScience 23, 101403 (2020)

21. A.M. Shaltout, K.G. Lagoudakis, J. van de Groep, S.J. Kim, J. Vučković, V.M. Shalaev, M.L. Brongersma, Science 365, $374(2019)$

22. C. Caloz, Z. Deck-Léger, IEEE Trans. Antennas Propagat. 68, $1583(2020)$

23. C. Caloz, Z. Deck-Léger, IEEE Trans. Antennas Propagat. 68, 1569 (2020)

24. X. Wang, A. Díaz-Rubio, H. Li, S.A. Tretyakov, A. Alù, Phys. Rev. Appl. 13, 044040 (2020)

25. L. Zhang, X.Q. Chen, S. Liu, Q. Zhang, J. Zhao, J.Y. Dai, G. D. Bai, X. Wan, Q. Cheng, G. Castaldi, V. Galdi, T.J. Cui, Nat. Commun. 9, 4334 (2018)

26. W. Kummer, A. Villeneuve, T. Fong, F. Terrio, IEEE Trans. Antennas Propagat. 11, 633 (1963)

27. A. Tennant, Electron. Lett. 33, 1768 (1997)

28. B. Chambers, A. Tennant, IEEE Antennas Propag. Mag. 46, $23(2004)$

29. L. Zhang, Z.X. Wang, R.W. Shao, J.L. Shen, X.Q. Chen, X. Wan, Q. Cheng, T.J. Cui, IEEE Trans. Antennas Propagat. 68, $2984(2020)$

30. L. Zhang, X.Q. Chen, R.W. Shao, J.Y. Dai, Q. Cheng, G. Castaldi, V. Galdi, T.J. Cui, Adv. Mater. 31, 1904069 (2019)

31. J.Y. Dai, J. Zhao, Q. Cheng, T.J. Cui, Light Sci. Appl. 7, 90 (2018)

32. J. Zhao, X. Yang, J.Y. Dai, Q. Cheng, X. Li, N.H.Q. Jun, C. Ke, G.D. Bai, S. Liu, S. Jin, A. Alù, T.J. Cui, Natl. Sci. Rev. 6, $231(2019)$

33. C. Zhang, J. Yang, L.X. Yang, J.C. Ke, M.Z. Chen, W.K. Cao, M. Chen, Z.H. Wu, J.F. Chen, Q. Cheng, T.J. Cui, Nanophotonics 9, 2771 (2020)

34. M. Liu, A.B. Kozyrev, I.V. Shadrivov, Phys. Rev. Appl. 12, $054052(2019)$

35. J.Y. Dai, W.K. Tang, J. Zhao, X. Li, Q. Cheng, J.C. Ke, M.Z. Chen, S. Jin, T.J. Cui, Adv. Mater. Technol. 52, 1900044 (2019)

36. J.Y. Dai, W. Tang, L.X. Yang, X. Li, M.Z. Chen, J.C. Ke, Q. Cheng, S. Jin, T.J. Cui, IEEE Trans. Antennas Propagat. 68, 1618 (2020)

37. H. Rajabalipanah, A. Abdolali, S. Iqbal, L. Zhang, T.J. Cui, 2020, arXiv:2002.06773 [physics.app-ph]

38. M. Minkov, Y. Shi, S. Fan, APL Photonics 2, 076101 (2017)

39. M. Liu, D.A. Powell, Y. Zarate, I.V. Shadrivov, Phys. Rev. X 8, 031077 (2018)

40. J. Kennedy, R.C. Eberhart, Proceedings of the 1997 IEEE International Conference on Systems, Man, and Cybernetics, Computational Cybernetics and Simulation, 1997, pp. 41044108, Vol. 5

41. A. Shaltout, A.V. Kildishev, V.M. Shalaev, Opt. Mater. Express 5, 2459 (2015)

42. Y. Hadad, D.L. Sounas, A. Alù, Phys. Rev. B 92, 100304 (2015)

43. Y. Hadad, J.C. Soric, A. Alù, Proc. Natl. Acad. Sci. U.S.A. 113, $3471(2016)$ 
44. X. Guo, Y. Ding, Y. Duan, X. Ni, Light Sci. Appl. 8, 123 (2019)

45. J.W. Zang, D. Correas-Serrano, J.T.S. Do, X. Liu, A. Alvarez-Melcon, J.S. Gomez-Diaz, Phys. Rev. Appl. 11, 054054 (2019)

46. A.E. Cardin, S.R. Silva, S.R. Vardeny, W.J. Padilla, A. Saxena, A.J. Taylor, W.J.M. Kort-Kamp, H.-T. Chen, D.A. R. Dalvit, A.K. Azad, Nat. Commun. 11, 1469 (2020)

47. W. Tang, J.Y. Dai, M. Chen, X. Li, Q. Cheng, S. Jin, K.-K. Wong, T.J. Cui, Electron. Lett. 55, 417 (2019)

48. W. Tang, X. Li, J.Y. Dai, S. Jin, Y. Zeng, Q. Cheng, T.J. Cui, China Commun. 16, 46 (2019)

49. W. Tang, M.Z. Chen, J. Yan Dai, Y. Zeng, X. Zhao, S. Jin, Q. Cheng, T.J. Cui, IEEE Wirel. Commun. 27, 180 (2020)

50. E. Basar, M. Di Renzo, J. De Rosny, M. Debbah, M. Alouini, R. Zhang, IEEE Access 7, 116753 (2019)

51. Q. Wu, R. Zhang, IEEE Commun. Mag. 58, 106 (2020)

52. W. Tang, J.Y. Dai, M.Z. Chen, K.-K. Wong, X. Li, X. Zhao, S. Jin, Q. Cheng, T.J. Cui, IEEE J. Sel. Area. Commun. 38, 2683 (2020)
53. M. Di Renzo, A. Zappone, M. Debbah, M. Alouini, C. Yuen, J.D. Rosny, S. Tretyakov, IEEE J. Sel. Area. Commun. 38, 2450 (2020)

54. G. Castaldi, M. Moccia, L. Zhang, A.Y. Hathaway, W. Tang, T.J. Cui, V. Galdi, Adv. Funct. Mater. (2020), https://doi. org/10.1002/adfm.202007620

55. S. Fallah, K. Rouhi, A. Abdolali, J. Phys. D Appl. Phys. 53, 085102 (2019)

56. H. Rajabalipanah, A. Abdolali, K. Rouhi, IEEE Trans. Emerg. Sel. Topics Circuits Syst. 10, 75 (2020)

57. J. Zhang, H. Zhang, W. Yang, K. Chen, X. Wei, Y. Feng, R. Jin, W. Zhu, Adv. Optical Mater. 8, 2000683 (2020)

58. J. Shabanpour, S. Beyraghi, A. Cheldavi, Sci. Rep. 10, 8950 (2020)

59. J. Li, Y. Zhang, J. Li, J. Li, Y. Yang, J. Huang, C. Ma, Z. Ma, Z. Zhang, L. Liang, J. Yao, Opt. Commun. 458, 124744 (2020)

60. L. Wang, Y. Zhang, X. Guo, T. Chen, H. Liang, X. Hao, X. Hou, W. Kou, Y. Zhao, T. Zhou, S. Liang, Z. Yang, Nanomaterials 9, 965 (2019)

Cite this article as: Lei Zhang, Jun Yan Dai, Massimo Moccia, Giuseppe Castaldi, Tie Jun Cui, Vincenzo Galdi, Recent advances and perspectives on space-time coding digital metasurfaces, EPJ Appl. Metamat. 7, 7 (2020) 